\title{
El uso de furosemida no prolonga la función renal residual en diálisis peritoneal
}

\author{
Francisco Cirera Segura, Jesús Lucas Martín Espejo, Antonia Concepción Gómez Castilla, María de los \\ Ángeles $\mathbf{O}$ jeda Guerrero
}

Unidad de Gestión Clínica Uro-Nefrológica. Hospital Universitario Virgen del Rocío. Sevilla

\section{Introducción:}

Uno de los cuidados de Enfermería en el programa de diálisis peritoneal (DP) es la preservación de la diuresis y de la función renal residual (FRR). Se suele recurrir a la administración de diuréticos, aunque es una medida controvertida. El objetivo principal fue comparar la diuresis y la FRR de los pacientes incidentes en DP y su evolución en el primer año de DP, en función del uso de diuréticos. El objetivo secundario fue valorar si los diuréticos puede mejorar el estado de volumen de nuestros pacientes.

\section{Material y métodos:}

Se realizó un estudio retrospectivo, comparativo, longitudinal y multicéntrico. La muestra estuvo compuesta por 40 pacientes. En una unidad se utilizaban diuréticos de forma protocolizada (grupo furosemida) y en la otra no (grupo control). En ambas se siguieron los mismos criterios de inclusión y exclusión. Se recogieron variables demográficas, relacionadas con el tratamiento, estado de volumen y los parámetros analíticos al inicio de DP, 6 y 12 meses. El análisis se realizó con el SPSS 19.

\section{Resultados:}

La muestra la compusieron 40 pacientes, el 57,5\% eran hombres. La edad media fue 56,45 + 16,2 años. EI $50 \%$ estaban en DP continua ambulatoria. La etiología de la Enfermedad Renal Crónica más prevalente fue nefritis intersticial $(22,5 \%)$, seguida de la vascular (20\%). Ningún paciente presentó efectos secundarios al uso de furosemida. Tras el estudio de las variables basales de ambos grupos, no se encontraron diferencias significativas excepto en el Kt/V renal $(p=0,004)$ y el potasio en orina $(p=0,007)$. La diuresis evolucionó de forma similar en ambos grupos durante el primer año en DP. Aunque siempre fueron superiores en el grupo furosemida, no llegó a ser significativo a los $6(p=0,160)$ ni a los 12 meses $(p=0,470)$. Estudiamos la evolución de los parámetros relacionados con la FRR y el estados de volumen, no hallando significación a lo largo del estudio en la diuresis $(p=, 836$ y $p=, 937)$, filtrado glomerular $(p=, 687$ y $p=332)$, peso $(p=, 942$ y $p=, 323)$, IMC $(p=, 861$ y $p=, 137)$ y ultrafiltración media $(p=, 796$ y $p=, 765$ ). El $40 \%$ de los pacientes del grupo control presentaron edemas a los 6 meses frente al $17,6 \%$ del grupo furosemida $(p=0,138)$. A los 12 meses fueron $13,3 \%$ vs $15,4 \% \quad(p=0,999)$. El grupo control utilizó mayor número de intercambios hipertónicos durante el primer año en DP. El grupo furosemida mostró presiones arteriales sistólicas superiores a los 6 y 12 meses ( $p=0,208$ y $p=0,347)$, lo mismo ocurrió con las diastólicas $(p=0,252$ y $p=0,5941)$. El grupo furosemida utilizó más hipotensores a los 6 y 12 meses $(p=0,082$ y $p=0,217$ ) sin significación. No encontramos diferencias en los parámetros analíticos sanguíneos ni en orina. El uso de furosemida no tuvo repercusión sobre la dosis de diálisis administrada.

\section{Discusión:}

La administración de dosis bajas de diuréticos en nuestra muestra, no ha mostrado diferencias significativas para la diuresis y la FRR durante el primer año en DP. A diferencia de otros estudios y quizás por las bajas dosis, el uso de diuréticos tampoco mejoró los parámetros relacionados con el estado de volumen. 


\section{Referencias Bibliográficas}

1. Rottemburgo J, Issad B, Gallego JL et al. Evolution of residual renal function in patients undergoing maintenance haemodialysis or continuous ambulatory peritoneal dialysis. Proc EDTA 1982; 19:397-402.

2. Lameire $\mathrm{N}$, Van Biesen $\mathrm{W}$. The impact of residual renal function on the adequacy of peritoneal dialysis. Perit Dial Int. 1997; 17(suppl 2):S102-S110.

3. James $F$, Medcalf $K$, Harris PG, Walls J. Role of diuretics in the preservation of residual renal function in patients on continuous ambulatory peritoneal dialysis. Kidney Int 2001; 59:1128-1133.

4. Lang S, Bergner A, Topfer M, Schiffl H. Preservation of residual renal function in dialysis patients: effects of dialysis-technique-related factors. Perit Dial Int 2001; 21(1):52-58.

5. Heimburguer 0 . Residual renal function, peritoneal transport characteristics and dialysis adequacy in peritoneal dialysis. Kidney Int 1996; 50(suppl 56):S47-S55.

6. Díaz-buzo JA, Gotch FA, Folden TI et al. Peritoneal dialysis adequacy: a model to assess feasibility with various modalities. Kidney Int. 1999; 55:2493-2501.

7. Guías SEN. Guías de práctica clínica en diálisis peritoneal de la SEN. Nefrología 2006; 26 (4).

8. Scarpioni I. Ballochi S, Bergonzi G et al. Highdose diuretics in continuous ambulatory peritoneal dialysis. Perit Dial Int. 1982;177-178.

9. Kam Tao Li P, Lun Cheng Y. Therapeutic options for preservation of residual renal function in patients on peritoneal dialysis. Perit Dial Int 2007; 27 (suppl 2):S158-S163.
10. Lysaght MJ, Vonesh EF, Gotch FA et al. The influence of dialysis treatment modality $\mathrm{ON}$ the decline of remaining renal function. ASAIO Trans. 1991; 37:598-604.

11. Canadá-usa (CANUSA) peritoneal dialysis study group. Adequacy of dialysis and nutrition in continuous peritoneal dialysis: association with clínical outcomes. J Am Soc. Nephrol 1996; 7:198207.

12. Li PK, Clown KM. The cost barrier to peritoneal dialysis in the developing world. An Asian perspective. Perit Dial Int 2001; 21(suppl 3):S307-S313.

13. Li PK, Szeto CC. Adequacy targets of peritoneal dialysis in the asian population. Perit Dial Int 2001; 21(suppl 3):S378-S383.

14. Hufnagel G, Michel C, Queffeulou G, Skhiri H, Damieri Mignon $F$. The influence of automated peritoneal dialysis on the decrease of residual renal function. Nephrol Dial Trasplant 1999; 14:12241228.

15. Tam, P. Peritoneal dialysis and preservation of residual renal function. Perit. Dial Int 2009; (suppl 2):S108-S110.

16. Menon MK, Naimark DM, Bargman JM, Vas SI, Oreopoulos DG. Long-term blood pressure control in a cohort of peritoneal dialysis patients and its association with residual renal function. Nephrol Dial Trasplant 2001; 16:2207-13.

17. Suda $T$, Hiroshige $K$, Ohta $T$, Watanabe $Y$, Iwamoto $\mathrm{M}$, Kanegae $\mathrm{K}$ et al. The contribution of residual renal function to overall nutritional status in chronic haemodialysis patients. Nephrol Dial Trasplant 2000; 15:396-401. 